\title{
De l'unification à nos jours : évolution du système éducatif dans les nouveaux Länder allemands
}

\section{Sandrine Lamer et Jörg Eschenauer}

\section{(2) OpenEdition \\ 1 Journals}

Édition électronique

URL : https://journals.openedition.org/ries/1956

DOI : 10.4000/ries. 1956

ISSN : 2261-4265

Éditeur

France Education international

\section{Édition imprimée}

Date de publication : 1 septembre 2002

Pagination : 133-143

ISBN : 978-285420-554-1

ISSN : 1254-4590

Référence électronique

Sandrine Lamer et Jörg Eschenauer, « De l'unification à nos jours : évolution du système éducatif dans les nouveaux Länder allemands », Revue internationale d'éducation de Sèvres [En ligne], 30 | septembre 2002, mis en ligne le 25 novembre 2011, consulté le 07 juillet 2021. URL : http:// journals.openedition.org/ries/1956 ; DOI : https://doi.org/10.4000/ries.1956

Ce document a été généré automatiquement le 7 juillet 2021.

(c) Tous droits réservés 


\title{
De l'unification à nos jours : évolution du système éducatif dans les nouveaux Länder allemands
}

\author{
Sandrine Lamer et Jörg Eschenauer
}

\section{Le contexte politique de 1989/90 ou le « vertige du tournant »}

1 La disparition du régime est-allemand après l'ouverture du mur de Berlin a été désignée par le terme die Wende (le tournant) afin de caractériser le changement radical de toute la vie politique, sociale et économique des Allemands de l'est. Comme tout virage pris à grande vitesse, ce tournant rapide s'accompagna de sensations de vertige qui sont à prendre en compte si l'on veut comprendre de l'intérieur l'expérience de la Wende. Quelle était l'atmosphère dans laquelle s'est opéré ce remplacement d'un modèle de société par un autre ?1

2 Le 6 mai 1989, le gouvernement d'Erich Honecker publiait les résultats des élections municipales: $98,85 \%$ des électeurs avaient voté pour les candidats du «Front National » du parti communiste. Ce chiffre inconcevable pour la population fissura la confiance des citoyens. La brèche fut accentuée par la perestroika de Mikhail Gorbatchev et sa fameuse phrase sibylline : «La vie punit tous les retardataires!» Les mois qui suivirent furent une course contre la montre durant laquelle tous les efforts furent déployés par l'État et ses adeptes pour sauver le système socialiste alors que les opposants s'évertuèrent à le faire tomber. La chute du mur le 9 novembre a rendu caduques tous ces efforts de l'ancienne élite communiste. La société civile estallemande se retrouva subitement - et sans être vraiment préparée - face aux exigences et aux défis d'un tournant historique. À partir de ce moment, tout alla très vite. Onze mois plus tard, l'Allemagne était unifiée. L'influence qu'exercèrent les représentants de la politique ouest-allemande sur les modalités de cette unification était telle que certains chercheurs ont avancé la thèse de la «colonisation de 
l'Allemagne de l'est par la RFA $»^{2}$. Seule la poésie est peut-être en mesure de faire éprouver cette nausée des Allemands de l'est qui, par ailleurs, « se sentaient comme des étrangers dans leur propre pays $»^{3}$. Dans ce contexte, le poème de Jürgen Nendza, intitulé "Logis ", est exemplaire car il reflète très bien non seulement cette expérience déroutante mais aussi une certaine autocritique ${ }^{4}:$ «La place/ entre/ les chaises/ nous voulions/l'occuper en accord/ être présents en observant/ les choses/ au jour/ le jour. / Nous ne trouvâmes/ aucune chaise. / Seulement cet/ espace vide/ rempli de voix"..

3 À cette déstabilisation identitaire de la société civile est-allemande correspondait entre 1990 et 1995 une Realpolitik des nouveaux décideurs politiques (venus de l'ouest ou non) qui, avec détermination, mettaient en place quasiment les mêmes structures scolaires qu'à l'ouest. Les rapports de forces étaient tels que l'est ne pouvait guère peser au début de la transformation. C'est depuis quelques années seulement que les Allemands de l'est redécouvrent avec une certaine fierté des atouts de leur culture politique jusqu'alors dévalorisée en bloc. Nous y reviendrons.

\section{D'un cadre institutionnel à l'autre}

4 Afin de mieux comprendre l'origine des difficultés qu'ont rencontrées les nouveaux Länder sur le plan éducatif depuis l'unification, il est nécessaire de faire un rappel ciblé sur les différences entre les deux Allemagnes qui préexistaient au « tournant $»^{5}$.

5 Contrairement au fédéralisme de l'ouest, c'est un système totalitaire et centralisé qui régissait la RDA dans son ensemble et plus particulièrement ses institutions d'éducation et de formation (einheitliches sozialistisches Bildung-ssystem). L'objectif central de l'éducation dans l'État socialiste allemand était de former l'individu à la vision socialiste du monde et de l'éduquer dans le seul intérêt de l'État.

6 En Allemagne de l'Est, il y avait un système d'école polyvalente unique : tout d'abord, la POS (polytechnische Oberschule), où un brevet de fin d'études à l'issue de la $10^{\mathrm{e}}$ classe (niveau $2^{\text {nde }}$ en France) devait être passé, puis la EOS (erweiterte Oberschule), école qui menait au baccalauréat $(A b i t u r)^{6}$. Des écoles d'élite existaient également pour les futurs sportifs professionnels, les musiciens ou les scientifiques. Enfin, des écoles spécialisées pour les enfants en difficulté pouvaient être une alternative au système traditionnel. L'orientation vers le brevet ou vers l'Abitur était décidée uniquement par les enseignants ${ }^{7}$ en fonction du profil des familles et des résultats scolaires de l'enfant.

7 L'enseignement était profondément marqué par l'idéologie communiste et il s'agissait pour les jeunes de se comporter dans le cadre des normes du seul modèle politique admis. Qui ne se pliait pas en ressentait immédiatement les conséquences par l'interdit: soit l'interdiction de participer à une manifestation solennelle, soit l'interdiction (quels que soient les résultats scolaires de l'enfant) d'aller à l'EOS ou encore l'interdiction pure et simple de suivre des études.

8 De même, cette «norme » était valable pour les parents : la République Démocratique Allemande s'était elle-même attribuée le titre de "premier État allemand des travailleurs et des paysans " (Erster deutscher Arbeiter- und Bauernstaat). Cela supposait que, pour des raisons idéologiques, seuls les enfants desdits « travailleurs et ouvriers " avaient droit d'accès à l'EOS ou au baccalauréat professionnel. Les enfants des « intellectuels» (et principalement des intellectuels non membres de la SED, du parti socialiste unifié de la RDA) se voyaient systématiquement limités à la POS. Ils devaient 
obtenir des dérogations pour, au mieux, passer un baccalauréat professionnel ou avoir des relations particulières avec le parti pour suivre le chemin privilégié !

9 L'idéologie se retrouvait également dans l'organisation du temps-libre des enfants autour de la journée scolaire. Chaque classe avait à préparer les manifestations-clés de l'État socialiste comme la parade du $1^{\mathrm{er}}$ mai par exemple. Par ailleurs, deux grandes organisations permettaient aux enfants de se retrouver les après-midi : pour les plus petits, la Ernst-Thälmann-Pionierorganisation, pour les plus grands, la Freie Deutsche Jugend (FDJ : « jeunesse allemande libre »), toutes deux organisations d'État visant, par le jeu, le sport et la vie en groupe, à former dès le plus jeune âge à l'esprit socialiste.

\section{Objectifs à l'aube des années 90}

C'est le 3 octobre 1990 qu'officiellement la RDA cessa d'exister. Son abolition avait cependant commencé bien avant cette date et les changements mis en place depuis 1989 sont impliqués dans un processus qui n'est pas encore achevé.

11 Le 16 février 1990 déjà, la Ständige Konferenz der Kultusminister (KMK, conférence permanente des ministres de l'éducation des Länder) se déclara prête à intégrer les Länder de l'est lorsqu'ils seraient reconstitués. Le 17 avril, les cinq Länder qui avaient eu leur légitimité jusqu'en 1952 furent rétablis sur le territoire de la RDA. Le 16 mai, une commission commune pour la question de l'éducation fut instaurée sous la direction des deux ministres de l'éducation et de la présidente de la KMK, Eva Rühmkorf. Suite à la réunification, l'enseignement, comme toutes les autres branches de l'administration, dut être entièrement reconçu. Mais l'application des nouvelles modalités ne pouvait se faire que progressivement. Quatre facteurs eurent et ont encore une influence notoire sur la mise en place des nouveaux dispositifs :

- le manque de temps et de ressources financières des Länder ;

- la décision puis l'instauration de nouveaux accords et contrats (par exemple avec la KMK, le fédéralisme supposant pour chaque nouvelle décision prise l'adhésion des 16 Länder) ;

- les problèmes liés au personnel ;

- la pression de l'opinion publique, qui attendait des résultats rapides et efficaces.

Les premiers changements instaurés dans le système éducatif de la RDA concernaient en priorité l'abolition de l'idéologie marxiste-léniniste jusqu'alors omniprésente. Dans cette phase d'esquisse du nouveau visage de l'est de l'Allemagne, la restructuration du système éducatif, scientifique et de la recherche apparaissait encore comme secondaire. Les contenus semblaient plus urgents. Dès la naissance de l'Allemagne unifiée, les textes du droit scolaire, mais aussi la méthodologie et la didactique de l'enseignement avaient connu leur première mutation. Les cours d'instruction civique et d'histoire avaient tout particulièrement été transformés dans leur substance même (les programmes avaient été entièrement revus), voire abolis.

13 Les établissements scolaires se trouvèrent également confrontés à la nécessité de s'ouvrir vers d'autres pays et avec d'autres objectifs que cela n'avait été le cas en RDA.

L'échec du système socialiste avait alors suffit à arguer des mérites du système ouestallemand dont les structures éducatives furent en 1990 reprises presque intégralement par l'est grâce à un système de parrainage entre les Länder: le MecklembourgPoméranie occidentale a été parrainé par le Schleswig--Holstein; la Thuringe par la Hesse, la Bavière, et la Rhénanie-Palatinat; la Saxe par le Bade-Wurtemberg et la 
Bavière ; la Saxe-Anhalt par la Basse-Saxe ; et enfin le Brandebourg par la Rhénanie-duNord-Westphalie.

Il est à noter que le Land de Berlin a totalement intégré Berlin-est dans sa structure. Berlin-est est le seul territoire de l'ex-RDA qui ait eu, sans transition aucune, un statut absolument identique à celui des anciens Länder. C'est la raison pour laquelle il est souvent question de «cinq" nouveaux Länder dans la littérature spécialisée qui ne mentionne pas la métropole, ou l'évoque en tant que cas particulier.

Les élections de la Diète des Länder (Landtagswahlen) d'octobre 1990 mirent cependant en évidence la volonté des nouveaux territoires d'obtenir au même titre que les anciens Länder une indépendance partielle dans leurs prises de décisions concernant le système éducatif. En cohérence avec les principes fondamentaux du fédéralisme allemand, ce droit leur a été octroyé.

\section{Évolution du système scolaire dans les nouveaux Länder dans les années 90 : continuité et changements}

17 Les changements opérés à l'est ne sont cependant pas le fruit d'une typologie contrastive. Le modèle ouest-allemand, certes dominant, n'a pas eu l'exclusivité de la modélisation des nouveaux Länder. Nous aborderons l'évolution à l'est sous deux aspects centraux du système éducatif, aujourd'hui encore au cœur des discussions politiques et mettant particulièrement en évidence l'avatar est-allemand : l'éducation pré-scolaire et l'encadrement extra-scolaire d'une part, l'enseignement général d'autre part.

\section{Éducation préscolaire et encadrement extrascolaire}

18 Le système socialiste ayant mis l'accent sur la famille et le droit au travail, nombreuses étaient en RDA les crèches et autres garderies qui permettaient aux femmes d'avoir des enfants tout en étant dans la vie active. Ces institutions, alors entièrement financées par l'État, furent remises en question en 1991. Mises en danger par le manque de fonds disponibles dans les caisses des Länder pour leur perpétuation, elles ont été l'objet de manifestations virulentes pour leur sauvegarde. Aujourd'hui, l'ouest de l'Allemagne jette un regard envieux vers ces structures, presque inexistantes dans les anciens Länder où, longtemps, les femmes se chargèrent intégralement de l'éducation de leurs enfants et restaient au foyer, situation qui disparait de plus en plus.

En 1991, c'est l'État fédéral qui avait financé la plus grande partie de ces établissements (30 \% des dépenses étaient supportées par lui). À partir de 1992, ce furent ensuite les fonds du mouvement de développement de l'est (Aufschwung Ost) qui furent employés, entre autres, à cet effet (5,5 milliards de DM).

Ces organisations sont aujourd'hui régies par la «loi de soutien de l'enfance et la jeunesse " (Kinder- und Jugendhilfegesetz) ${ }^{8}$ et financées par différents représentants (contrairement à l'époque socialiste): des organisations privées, communales ou de communautés religieuses. Mais à l'est, ce sont les institutions communales qui 
dominent, alors qu'à l'ouest, les garderies privées occupent $25 \%$ du marché, les places publiques étant monnaie rare...

21 Chaque nouveau Land formule cependant différemment le droit à cette forme d'éducation. Le Brandebourg signale que tout enfant a droit, jusqu'à son entrée à l'école obligatoire, à une place dans une garderie, "dans la mesure d'un justificatif de nécessité ». Une famille dont un membre serait en mesure de garder l'enfant n'aurait donc pas droit à une place de garderie. En Mecklenbourg-Poméranie occidentale, il n'est pas stipulé littéralement que tout enfant doit avoir la possibilité d'être mis en crèche ou garderie, mais le préambule du texte de loi met en évidence le «droit de l'enfant à un soutien du Land». C'est en Saxe que les textes sont les plus clairs: ils indiquent que tout enfant à partir de trois ans peut obtenir une place en Kindergarten jusqu'à son entrée en école élémentaire. Les représentants de ces institutions se doivent de mettre en place les structures nécessaires à l'accueil des enfants et leur éducation. En Thuringe, c'est à partir de deux ans et six mois que les enfants peuvent être pris en charge par le Kindergarten.

En Saxe-Anhalt enfin, la prise en charge de l'éducation des enfants en pré-scolarité ou en situation extrascolaire est de loin la plus étendue. Leur droit à une place dans des institutions à cet effet va de la crèche ou garderie à la place en jardin d'enfants, mais il comprend aussi les ateliers en dehors des cours pour les jeunes jusqu'à leur quatorzième année accomplie. Cette réglementation a pour atout de reprendre une structure socialiste, en soi fort bien conçue pour les familles et les jeunes, sans son contexte idéologique. C'est vers ce type de fonctionnement qu'aimeraient se tourner, à partir de 2003, d'autres nouveaux Länder comme la Saxe par exemple, qui revient de plus en plus sur des conceptions socialistes dépolitisées (comme l'éducation d'une élite ou l'éducation continue).

En 1997, le nombre de places disponibles par rapport à la demande allait de 96,2 \% en Thuringe à 163,4 \% en Saxe-Anhalt. Cette surenchère de places s'explique, d'une part, par les départs en masse des jeunes familles vers l'ouest, qui perdurent jusqu'en 2002, d'autre part, la chute draconienne des natalités 9 face à l'incertitude de l'avenir.

Depuis 1997, des plans de fermeture systématique des garderies sont établis. Le nombre de ces institutions a déjà été réduit de $25 \%^{10}$. Pourtant, à partir de 2000 , on voit se rétablir un mouvement de sauvegarde des centres et, pour l'instant, les fermetures sont stoppées.

\section{Enseignement général}

La réorganisation de l'enseignement primaire et secondaire s'est heurtée à de multiples difficultés, brièvement évoquées plus haut, qu'il s'agit ici d'extrapoler. Trois points devaient être traités en urgence : la nouvelle structure qui remplacerait la POS et EOS ; les contenus et méthodes de l'enseignement; la formation des enseignants. Chaque Land, dans le contexte qui était le sien, a réalisé ces changements à sa manière. 


\section{Évolution de la structure du système éducatif}

26 À première vue, il semblerait que le système en vigueur à l'ouest ait prévalu. C'est pourquoi nous mettrons particulièrement l'accent sur les spécificités des nouveaux Länder par rapport aux anciens.

$\mathrm{Au}$ centre de la discussion de la restructuration se sont trouvées d'abord les Gesamtschulen ${ }^{11}$, comme cela avait été le cas quelques années auparavant dans les anciens Länder. Elles présentaient des similitudes avec le système de la POS et EOS et auraient donc supposé un moindre bouleversement de l'organisation générale. Par ailleurs, elles permettaient de faire face au problème de rayonnement de l'école en superficie par rapport à une population en décroissance rapide. Leur mise en place semblait donc à priori être la plus simple à réaliser. Cependant le Brandebourg est le seul Land ayant défendu aussi vivement cette structure, dont la présence y est plus importante que le Gymnasium (elles représentent, à la fin des années 90, plus de $50 \%$ des établissements secondaires ${ }^{12}$ ). En Mecklenbourg-Poméranie occidentale, quelques Gesamtschulen trouvent leur place alors qu'en Saxe et en Saxe-Anhalt, cette structure n'est absolument pas représentée. Aujourd'hui, le Brandebourg prévoit d'abolir petit à petit les Gesamtschulen et de les remplacer par les Gymnasien, qui se sont imposés à plus de 30 \% dans les autres Länder depuis 1991.

Par ailleurs, malgré la pression exercée sur les décideurs des nouveaux Länder et en dépit des droits limités dont ils disposaient, ils ont tout de même fait le choix de certaines alternatives pour ne pas tomber dans les erreurs connues à l'ouest. Ceci concerne surtout les Hauptschulen.

Le système démocratique fédéral allemand suppose un choix d'orientation à l'issue de l'école primaire. À l'ouest, trois types d'écoles sont possibles : le Gymnasium (qui mène à l'Abitur), la Realschule (qui mène à la "maturité moyenne»: Mittlere Reife), la Hauptschule (qui mène à un brevet de fin d'études). L'évolution des sociétés industrielles exigeant de plus en plus de diplômes spécialisés, la Hauptschule est devenue une forme de Restschule (l'école pour le reste des élèves, ceux qui n'ont pas eu de place ailleurs), dans laquelle de grands problèmes de discipline apparaissent, ainsi que des inquiétudes liées au devenir des élèves qui la quittent.

Pour pallier ce problème, les Länder de l'est ont choisi une formule d'alternative : l'école secondaire à deux profils - une forme de «transformation expérimentale [du système scolaire allemand] $»^{13}$. Le premier profil est celui du Gymnasium, qui a connu un véritable succès chez les parents. Quant au second profil, il s'intitule Realschule dans le Brandebourg et le Mecklenbourg-Poméranie occidentale, Mittelschule en Saxe, Sekundarschule en Saxe-Anhalt, Regelschule en Thuringe. Chacun de ces Länder (excepté le Brandebourg) offre cependant, à l'intérieur des «seconds profils", la possibilité de s'orienter vers un niveau de Hauptschule, afin que les élèves en difficulté ne soient pas trop déstabilisés. Ces trois nouveaux modèles allemands de Saxe, Saxe-Anhalt et Thuringe ont fait leurs preuves et les élèves sortant trouvent une place de plus en plus reconnue sur le marché du travail. Les exigences du Gymnasium mettent trop d'élèves en échec, et le vent tourne dans le sens d'une préférence pour un meilleur diplôme de Mittelschule ou Regelschule plutôt qu'un mauvais Abitur après lequel les portes se ferment. 
31 Un autre point fort hérité de la RDA consiste dans les douze années de scolarité jusqu'à l'obtention du diplôme de fin de second cycle. La dynamique européenne n'est pas sans influencer une évolution vers une plus grande concentration de la scolarité. Si la tradition de l'ouest est de mener les élèves à l'Abitur par un cursus de 13 ans, cette tendance évolue vers une réduction à 12 ans, déjà instaurée en Sarre et en discussion dans le Bade-Wurtemberg. Les élèves allemands sont en effet en moyenne plus âgés que les autres élèves européens lorsqu'ils terminent leurs études, d'où une réflexion actuelle sur les possibilités et moyens à mettre en œuvre pour remédier à cet état de fait. Les changements commenceraient déjà à l'école. Cette réflexion s'accompagne du constat que la "durée moyenne de vie» du savoir diminue de plus en plus rapidement ${ }^{14}$. Il est donc nécessaire de mettre les savoirs enseignés à l'école le plus rapidement possible en application dans le cadre des études. Le MecklenbourgPoméranie occidentale avait mis en place en 2000 l'Abitur en 13 ans afin de mieux se calquer sur le modèle du Schleswig-Holstein et d'appliquer la loi de la KMK réglementant le nombre d'heures minimum pour se présenter aux examens. Afin d'alléger les journées des élèves, une année supplémentaire avait alors été introduite, mais supprimée de nouveau dès la rentrée 2002/2003. Si bien qu'en 2007/2008, dans ce Land, certains élèves passeront l'Abitur après 12 ans, d'autres après 13 ans d'école !

Réduire le nombre d'années de scolarité signifie concentrer davantage les enseignements. En effet, il n'est pas question de réduire les programmes comme les années! Dans les quatre nouveaux Länder concernés par une scolarité plus concentrée, les cours se poursuivent souvent les après-midi (ils s'échelonnent entre 7 heures et 17 heures; mais en moyenne, les élèves ont cours jusqu'à 15 heures). L'image des couloirs de l'école allemande dépourvus de toute vie à partir de 13 heures n'est donc plus vraie aujourd'hui sur l'ensemble du pays et semble être amenée à disparaître peu à peu. Au contraire même, il est de plus en plus question de remettre en place des activités extra-scolaires dans l'enceinte de l'école après les cours, comme cela était de coutume en RDA. L'aide aux devoirs ou des ateliers divers (Arbeitsgemeinschaften) permettent aux enfants non seulement de développer leurs compétences dans d'autres domaines que ceux uniquement liés aux programmes scolaires, mais aussi d'occuper de façon constructive leur temps libre. La Saxe et le Mecklenbourg-Poméranie occidentale investissent actuellement beaucoup dans ce domaine.

33 Enfin, la structure d'ensemble du paysage politique sur le plan de l'éducation évolue depuis six ans par secousses. L'évolution démographique est telle qu'un plan de fermeture systématique des établissements scolaires a été institué. À titre d'exemple, la Saxe comptait 770000 élèves à la rentrée 1994/1995. En 2001/2002, ils n'étaient plus que 620 000. En 2010, 420000 enfants seulement seront inscrits à l'école. La situation est due, nous l'avons vu, à l'inconnu de l'après-unification et à la migration en masse des jeunes familles vers l'ouest, qui se poursuit jusqu'au XXI siècle, la situation économique et sociale des nouveaux Länder étant très fragile. Le Brandebourg et le Mecklenbourg-Poméranie occidentale sont les plus concernés par ces départs d'une population active et jeune. Les premières structures touchées furent les écoles primaires. Depuis 2000, le secondaire accueille la vague de la "génération-tournant ». En moyenne, 30 \% des établissements ont été fermés. Afin de ne pas provoquer trop de licenciements, différents accords ont été conclus dans les Länder avec le personnel enseignant. Des pré-retraites avec prime de départ au temps partiel (les instituteurs sont, selon les Länder, employés et payés de $50 \%$ en Thuringe à $76 \%$ en Saxe) ou aux 
accords avec les Länder limitrophes (Thuringe, Hesse), tous les moyens sont mis en œuvre pour que les réformes soient acceptées par compromis. Mais si l'on considère que dans les nouveaux Länder, les enseignants ont un statut d'employés et non de fonctionnaires, sont rémunérés à $90 \%$ des salaires des employés des services publics de l'ouest et donnent jusqu'à 27 heures de cours (en Saxe) par semaine, soit jusqu'à 6 heures hebdomadaires de plus que dans d'autres Länder de l'ouest - sans compter les remplacements obligatoires -, il sera aisé de comprendre que cette situation crée une atmosphère de désenchantement parmi les enseignants qui pour la plupart, ont fait preuve de beaucoup de courage depuis l'unification.

\section{De nouveaux contenus d'enseignement} travail de projet qui est prôné par l'ouest. Une remise en question des méthodes d'enseignement a donc été exigée par la KMK, mais pas toujours réalisée : «les structures scolaires et les programmes sont bien plus simples à modifier que le cours en lui-même - un comportement des enseignants lié aux habitudes prises persiste souvent face à ces conceptions nouvelles, tout aussi persuasives qu'elles soient $»^{15}$.

L'évaluation est enfin un thème au centre des discussions. Le fédéralisme allemand rend en effet difficile la comparaison des bulletins de notes ou diplômes et freine la mobilité. C'est pourquoi il est nécessaire de contrôler les critères d'évaluation. Parmi les nouveautés, les notes générales concernant l'élève sont également un héritage de la RDA et s'imposent de plus en plus en Allemagne: sur son bulletin, l'élève de la Sekundarstufe I a non seulement les résultats obtenus dans les différentes disciplines, mais aussi une estimation de son attitude générale en cours, issue d'une moyenne des notes données par les enseignants. Ces notes mentionnées en haut du bulletin (Kopfnoten) vont de 1 à 4 (1 étant la meilleure note), et évaluent: l'ordre, le comportement, l'assiduité, la participation (active ou non). D'après un sondage, ce système d'évaluation a rencontré $80 \%$ d'approbation dans l'ensemble de l'Allemagne ${ }^{16}$. Il se pourrait donc fort que l'ouest intègre prochainement ces notes dans ses bulletins scolaires, d'autant plus que les chefs d'entreprises les apprécient lorsqu'il s'agit, par exemple, de proposer des stages ${ }^{17}$ ou des places d'apprentissage. À l'heure actuelle, tous 
les nouveaux Länder ont rétabli cette notation s'ils ne l'avaient pas conservée depuis 1990.

\section{La formation des enseignants}

L'École de l'est se trouvait donc, au début des années 1990, face à la situation paradoxale d'une nécessité de changement radical des structures, des méthodes et des contenus de l'enseignement, tout en travaillant avec le personnel de l'éducation qui avait fait ses preuves en RDA.

Plusieurs années durant, les enseignants de l'ex-RDA se sont battus afin que leurs diplômes soient acceptés au niveau du Zweites Staatsexamen, examen nécessaire à la qualification en tant qu'enseignant du second degré. Les études en RDA étant de moindre durée, les autorités ne reconnaissaient pas le diplôme d'enseignant comme un parcours fini, ce qui avait des conséquences notoires sur le salaire mensuel et sur les retraites. Aujourd'hui, la réglementation de reconnaissance est mise en place.

41 Il est même question de réduire la période de formation théorique des professeurs et de renforcer dès le début des études la pratique des stages, comme c'était de coutume en RDA.

\section{Bilan et enjeux actuels}

Pour changer le fonctionnement d'un système scolaire, on ne peut pas lui prescrire une cure de repos ni une remise en état en douceur. Ce type de patient est obligé de continuer à travailler et les médecins ne peuvent intervenir qu'en respectant les rythmes de l'activité permanente du malade. Si on prend en compte l'énorme chantier que représentait le système politique est-allemand dans sa totalité en 1990, on doit reconnaître que la transformation du système éducatif s'est globalement passée d'une façon satisfaisante. Certes, les Länder de l'est durent reprendre grosso modo les modèles de leurs partenaires à l'ouest. En 1990, on n'avait ni le temps, ni les moyens, ni les idées assez claires pour mettre en place des structures et des démarches pédagogiques innovatrices. Mais quel autre pays en Europe a montré ces dernières années un goût prononcé pour des réformes audacieuses dans le domaine éducatif? Il fallait être pragmatique en créant les bases juridiques indispensables en droit scolaire afin de surmonter au plus vite les énormes problèmes démographiques, idéologiques, financiers et pédagogiques du début de la décennie. À partir de 1995, chaque Land estallemand avait terminé le travail législatif dans les secteurs essentiels de la société ${ }^{18}$ et les Länder est-allemands ont alors commencé à s'affirmer plus fermement face aux anciens Länder en développant leurs propres réformes: arrêt de la fermeture des crèches ou des garderies; abandon de la Hauptschule et création de l'école secondaire à deux profils; réduction du temps de scolarité et d'études universitaires; introduction d'un choix entre des cours d'éthique et des cours de religion; système de notation valorisant les vertus de bases (Primärtugenden) comme l'assiduité, l'ordre, la participation et le comportement.

43 Le débat public sur la question de l'école est donc, aujourd'hui, de moins en moins marqué par un écart de qualité est-ouest. Les deux parties de l'Allemagne se trouvent plutôt confrontées à un nouveau défi commun : l'étude PISA. Les mauvais résultats des 
élèves allemands ont provoqué un véritable tremblement de terre dans l'opinion publique. Les seize ministres de la KMK doivent réagir d'une manière concertée après la publication des résultats détaillés qui est prévue pour la fin de cette année.

C'est une forme d'ironie de l'histoire: les Allemands de l'est semblent en effet être mieux préparés aujourd'hui aux changements qui s'imposent au système éducatif allemand. Les nouveaux objectifs de la politique éducative seront certainement axés sur la rigueur et l'exigence, sur une nouvelle «culture de l'effort $»^{19}$. Une certaine discipline, développée à l'époque de la RDA exclusivement au service de l'idéologie marxiste, est aujourd'hui réhabilitée en tant que vertu de base nécessaire à toute formation de l'individu capable d'assumer son autonomie. À l'ouest, on dénonce par contre les effets pervers d'une Kuschelpädogogik (pédagogie des câlins), c'est-à-dire d'une pédagogie molle, trop permissive, promulguée par la génération 68 .

L'école allemande qui fut un modèle pour ses voisins ${ }^{20}$, se replie aujourd'hui de plus en plus dans une attitude d'auto-critique. Les résultats de l'étude PISA n'ont fait que renforcer ce mouvement de dévalorisation du système éducatif allemand actuel et surtout de sa perception par l'opinion publique. Les nouveaux Länder n'ont aucun complexe à avoir, ils se trouvent comme les anciens devant le même enjeu d'adaptation du système scolaire au monde qui change : comment préparer les élèves à la vie et au travail dans une société d'information et de savoirs perpétuellement rénovés? Comment mesurer et assurer la qualité de l'enseignement de chaque école et d'un système scolaire dans son ensemble? Comment garantir l'efficacité de la formation permanente des enseignants?

Le président de la RFA, Johannes Rau, a cité l'année dernière, dans un discours consacré à l'éducation, le chancelier Ludwig Erhard qui disait le 19 octobre 1963 devant le Bundestag: "Sans renforcement de l'investissement intellectuel, l'Allemagne serait amenée à reculer face à d'autres pays industriels et civilisés. Cela mettrait en jeu non seulement le développement économique et la prospérité de notre pays, mais aussi sa sécurité sur le plan social. Gouvernement fédéral et Länder doivent agir ensemble pour aborder résolument une mission commune de haute importance. Le peuple allemand doit prendre conscience que la tâche de l'éducation et celle de la recherche pour nos descendants sont de la même ampleur que la question sociale pour le XIX ${ }^{\mathrm{e}}$ siècle ${ }^{21}$. Selon Johannes Rau, l'Allemagne se trouve aujourd'hui, à nouveau, devant un devoir impératif de réforme du système scolaire, comme dans les années soixante. Mais, poursuit-il, «à l'époque, la discussion s'est transformée finalement en un débat stérile chargé d'idéologie sur l'organisation de meilleures structures scolaires et universitaires. Jadis déjà, ce n'était pas bon. Aujourd'hui nous devons mieux réussir ${ }^{22}$. Une chose au moins est sûre : face à ce défi, il n'y a plus de différence, dix ans après l'unification, entre l'est et l'ouest de l'Allemagne. 


\section{NOTES}

1. Le résumé suivant se base en partie sur la publication suivante : H.-W. Fuchs/L.R. Reuter/D. Smoczynski: Chronik bildungs- und wissenschaftspolitischer Entwicklungen und Ereignisse in Ostdeutschland 1989 bis 1996, Beiträge aus dem Fachbereich Pädagogik der Universität der Bundeswehr Hamburg (Heft 1), 1997.

2. F. Vilmar/G. Guittard: La face cachée de l'unification allemande, Les éditions de l'Atelier, Paris 1999.

3. Frankfurter Rundschau du 9.9.1991.

4. K.O. Conrady (Hg.) : Vom einen Land und vom andern. Gedichte zur deutschen Wende, Suhrkamp, Frankfurt/M./Leipzig 1993, p. 81 (traduction des auteurs).

5. Il est bien entendu qu'il ne s'agit pas ici de présenter de façon exhaustive le fonctionnement du système politique de la RDA. Certains éléments de cette politique ont été choisis afin de mettre en évidence les grandes différences des deux systèmes avant et après l'unification dans la partie est de l'Allemagne.

6. Jusqu'en 1983, la POS se divisait à partir de la $9^{\mathrm{e}}$ classe vers l'EOS, l'école professionnelle avec Abitur, ou vers la formation professionnelle seule. Ce n'est qu'à partir de 1983 que la POS allait jusqu'à l'issue de la $10^{\mathrm{e}}$ classe.

7. Ces enseignants se devaient de rendre régulièrement visite aux parents pour parler de l'élève dans son contexte familial.

8. Cf. le tableau de présentation des droits dans les crèches et de l'organisation de garderies de jour du ministère de la famille, des personnes âgées, des femmes et de la jeunesse: Bundesministerium für Familie, Senioren, Frauen und Jugend 1994, p. 497, tableau V.7.11.

9. Entre 1989 et 1992, les naissances ont diminué de plus de moitié.

10. Les chiffres actuels nous ont été fournis lors d'entretiens téléphoniques avec les attachés de presse des Ministères de l'éducation des nouveaux Länder, que nous tenons à remercier pour leur collaboration.

11. Pour plus de renseignements sur le système scolaire de la RFA, cf. Traditions et transformations, Le système d'éducation en République fédérale d'Allemagne, Max-Planck-Institut für Bildungsforschung, Economica, Paris, 1991.

12. Cf. Hans Döbert: «Schule in Ostdeutschland zwischen zwei Transformationsprozessen » in Transformation in der Ostdeutschen Bildungslandschaft, Leske und Budrich, Opladen 2002, p. 37.

13. Hans Döbert, op. cit., p. 39.

14. D’après M. Schattschneider, attaché de presse du Land Mecklenbourg-Poméranie (mars 2002).

15. Klaus-Jürgen Tillmann, cité par Axel Gehrmann dans «Gewandelte Lehrerrolle in Ost und West? Erste Ergebnisse aus vier Befragungen (1994 - 1996 - 1998 - 1999)» in "Schule in Ostdeutschland zwischen zwei Transformationsprozessen ", Transformation in der Ostdeutschen Bildungslandschaft, Leske und Budrich, Opladen 2002, p. 63.

16. D'après Stefan Große, attaché de presse du Ministère de l'éducation et de la culture de Saxe (interview de mars 2002).

17. Il faut savoir qu'en Allemagne, tout élève est tenu, même dans l'enseignement général, de faire un stage en entreprise. Ce stage a souvent lieu en $9^{\mathrm{e}}$ ou $10^{\mathrm{e}}$ classe, selon l'organisation interne des Länder, voire des établissements scolaires eux-mêmes.

18. Cf. A. Thumfart: Die politische Integration Ostdeutschlands, Suhrkamp, Frankfurt/M. 2002, p. 420-599.

19. Johannes Rau, président de la RFA, lors d'un discours récent.

20. Cf. «L'école allemande, un modèle ?» (dossier), Cahiers Pédagogiques №359, décembre 1997, p. 8-59. 
21. www.bundespraesident.de/dokumente/Rede/ix_45939.html.

22. Idem.

INDEX

Mots-clés : système éducatif

Index géographique : Allemagne

\section{AUTEURS}

\section{SANDRINE LAMER}

Chargée de programme à l'unité des assistants de langues et stages linguistiques (pays germanophones (Allemagne, Autriche, Suisse) et Russie) au CIEP.

\section{JÖRG ESCHENAUER}

Docteur en sciences politiques, directeur pédagogique des sections internationales du collège et du lycée de Sèvres. 